PUPT-1891

ITEP-TH-48/99

NSF-ITP-99-27

YCTP-P24-99

hep-th/9909109

\title{
An Orbifold of Type 0B Strings and Non-supersymmetric Gauge Theories
}

\author{
Igor R. Klebanov, ${ }^{1}$ Nikita A. Nekrasov ${ }^{2}$ and Samson L. Shatashvili ${ }^{3 \dagger}$ \\ 1,2 Joseph Henry Laboratories, Princeton University, Princeton, New Jersey 08544 \\ 2 Institute for Theoretical and Experimental Physics, 117259 Moscow, Russia \\ 3 Department of Physics, Yale University, New Haven, CT 06520
}

\begin{abstract}
We study a $\mathbb{Z}_{2}$ orbifold of Type $0 \mathrm{~B}$ string theory by reflection of six of the coordinates (this theory may also be thought of as a $\mathbb{Z}_{4}$ orbifold of Type IIB string theory by a rotation by $\pi$ in three independent planes). We show that the only massless mode localized on the fixed fourplane $\mathbb{R}^{3,1}$ is a $U(1)$ gauge field. After introducing D3-branes parallel to the fixed fourplane we find non-supersymmetric non-abelian gauge theories on their worldvolume. One of our results is that the theory on equal numbers of electric and magnetic D3-branes placed at the fourplane is the $\mathbb{Z}_{4}$ orbifold of $\mathcal{N}=4$ supersymmetric Yang-Mills theory by the center of its R-symmetry group.
\end{abstract}

$09 / 99$

$\dagger$ On leave of absence from Steklov Mathematical Institute, St.Petersburg, Russia 


\section{Introduction}

The discovery of AdS/CFT correspondence [1,2,3] has led to new insights into four dimensional gauge theories. In particular the results on D3-branes near orbifold singularities [⿴囗十 naturally lead to quotients of the basic $\mathcal{N}=4$ duality by discrete subgroups of the $S U(4)$ R-symmetry [5,60. A closely related development has been the study of gauge theories on D3-branes of Type 0B theory, which is an NSR string with the non-chiral GSO projection $(-1)^{F+\tilde{F}}=1$ which breaks all spacetime supersymmetry [7]. Type 0B string can also be studied in the GS framework, as we show below.

The Type $0 \mathrm{~A}$ and $0 \mathrm{~B}$ models have twice as many massless RR fields as their Type II cousins, hence they also possess twice as many D-branes [8]. For example, since Type 0B spectrum has an unrestricted 4-form gauge potential, there are two types of D3-branes: those that couple electrically to this gauge potential, and those that couple magnetically. Very importantly, the weakly coupled spectrum of open strings on Type 0 D-branes does not contain tachyons after the GSO projection $(-1)^{F_{\text {open }}}=1$ is implemented [8, 9, 10]. Thus, gauge theories living on such D-branes do not have obvious instabilities at weak coupling. This suggests via the gauge field/string duality that the bulk tachyon instability of Type 0 theory may be cured as well [8,11].

The simplest example of AdS/CFT duality in a Type 0 context follows from considering $N$ electric D3-branes coincident with $N$ magnetic ones [11]. For such a stack the net tachyon tadpole cancels so that there exists a classical solution with $T=0$. In fact, since the stack couples to the selfdual part of the 5 -form field strength, the Type 0B 3-brane classical solution is identical to the Type IIB one. Taking the throat limit suggests that the low-energy field theory on $N$ electric and $N$ magnetic D3-branes is dual to the $\operatorname{Ad} S_{5} \times S^{5}$ background of Type 0B theory and is therefore conformal in the planar limit [11]. This theory is the $U(N) \times U(N)$ gauge theory coupled to six adjoint scalars of the first $U(N)$, six adjoint scalars of the second $U(N)$, and Weyl fermions in the bifundamental representations - four in the $(\mathbf{N}, \overline{\mathbf{N}})$ and four in the $(\overline{\mathbf{N}}, \mathbf{N})$ (the $U(1)$ factors decouple in the infrared). This theory is a $\mathbb{Z}_{2}$ projection of the $\mathcal{N}=4 U(2 N)$ gauge theory [11]. The $\mathbb{Z}_{2}$ is generated by $(-1)^{F_{s}}$, where $F_{s}$ is the fermion number, together with conjugation by $\left(\begin{array}{cc}I & 0 \\ 0 & -I\end{array}\right)$ where $I$ is the $N \times N$ identity matrix. This is related to the fact that Type 0 string theories may be viewed as $(-1)^{F_{s}}$ orbifolds of the corresponding Type II theories [7]. In [12] it was pointed out that $(-1)^{F_{s}}$ is identified with an element of the center of the $S U(4)$ R-symmetry, hence this $\mathbb{Z}_{2}$ projection of the $\mathcal{N}=4$ theory belongs to the class studied in [5,6]. In particular this observation explains why the theory is conformal in the large $N$ limit (this follows from the arguments of [13]), and also why the dual string theory 
background is $A d S_{5} \times S^{5}$ - the reason being that the orbifold group $\mathbb{Z}_{2}$ belongs to the spin cover of the Lorentz group and projects to identity in the rotation group. Therefore the geometry is not affected by the quotienting.

In view of this observation it is of further interest to study the quotient of the maximally supersymmetric theory by the full center $\mathbb{Z}_{4}$ of $S U(4)$ [12]. This theory has

- the gauge group $U(N)^{4}$ coupled to a chiral field content:

- four quadruples of bi-fundamental fermions transforming in $\left(\mathbf{N}_{\mathbf{i}}, \overline{\mathbf{N}}_{\mathbf{i}+1}\right), \mathbf{i}=0,1,2,3$; $4 \equiv 0$,

- four sextets of bi-fundamental scalars in $\left(\mathbf{N}_{\mathbf{i}}, \overline{\mathbf{N}}_{\mathbf{i}+2}\right)$.

Can this gauge theory be embedded into string theory? Since the generator of $\mathbb{Z}_{4}$ changes the sign of the six scalar fields in the $\mathcal{N}=4$ multiplet, a good guess is that this theory occurs on D3-branes of Type 0B theory orbifolded by reflection of six spacetime coordinates [12]. In this paper we confirm this guess and also derive some further properties of this $\mathbb{Z}_{2}$ orbifold of Type 0B theory (more general orbifolds of Type 0B were studied in [14, 15]). One interesting result is that the only massless mode on the fixed fourplane $\mathbb{R}^{3,1}$ is a photon. After D3-branes are added, this $U(1)$ gauge field couples to the the theory on the D3-branes, and we comment on some features of this coupling. We regard this as an interesting non-supersymmetric analogue of what happens in orbifolding Type IIB theory by reflection of four of the coordinates. There we find a tensor theory on the fixed six-plane $\mathbb{R}^{5,1}$.

\section{The field theories.}

Our goal is to study a certain $\mathbb{Z}_{4}$ orbifold of Type IIB string theory 1 Let us consider the orbifold of Type IB theory by the $\mathbb{Z}_{2}$ reflection of six out of ten space-time coordinates:

$$
X^{M} \rightarrow-X^{M}, \quad M=4, \ldots, 9 .
$$

Under the corresponding breaking of the Lorentz group $S O(1,9) \rightarrow S O(1,3) \times S O(6)$ the ten-dimensional Mayorana-Weyl spinors decompose as:

$$
\mathbf{1 6}=\mathbf{2}_{\mathbb{C}}^{S O(1,3)} \otimes \mathbf{4}^{S O(6)}
$$

The transformation (2.1) acts on the space-time spinors via multiplication by $i$ and this $\mathbb{Z}_{2}$ becomes actually $\mathbb{Z}_{4} \in S U(4)=\operatorname{Spin}(6)$.

1 Notice that there are many possibilities of embedding the group $\mathbb{Z}_{4}$ into the global symmetry group of the Type IIB string theory. We consider the embedding which does not break the global symmetry. 
The generator of this $\mathbb{Z}_{4}$ is a rotation by $\pi$ in the 45,67 and 89 planes, exp $\pi i\left(J_{45}+\right.$ $\left.J_{67}+J_{89}\right)$. This group has a $\mathbb{Z}_{2}$ subgroup generated by $\exp 2 \pi i\left(J_{45}+J_{67}+J_{89}\right)$. If we orbifold the Type IIB string theory by this $\mathbb{Z}_{2}$ then we get the Type 0B string. Indeed, it is known that $0 \mathrm{~B}$ is an orbifold of $\mathrm{IB}$ by $(-1)^{F_{s}}$ where $F_{s}$ is the spacetime fermion number [7], and $\exp 2 \pi i\left(J_{45}+J_{67}+J_{89}\right)$ acts on all fields as $(-1)^{F_{s}}$. The subsequent orbifolding of Type $0 \mathrm{~B}$ string by $\exp \pi i\left(J_{45}+J_{67}+J_{89}\right)$ is actually a $\mathbb{Z}_{2}$ operation because the theory has no fermions in its spectrum. Thus, the theory we are studying may also be thought of as a $\mathbb{Z}_{2}$ orbifold of Type $0 \mathrm{~B}$ string by reflection of six coordinates (2.1).

The same conclusion applies to the field theory on D3-branes. Take $N$ D3-branes placed at the fixed point of the discrete group. The theory induced on the world volume of these branes will have four $U(N)$ gauge groups, four quadruples of bi-fundamental fermions transforming in $\left(\mathbf{N}_{\mathbf{i}}, \overline{\mathbf{N}}_{\mathbf{i}+1}\right)$, four sextets of bi-fundamental scalars in $\left(\mathbf{N}_{\mathbf{i}}, \overline{\mathbf{N}}_{\mathbf{i}+2}\right)$, $\mathbf{i}=0,1,2,3 ; \mathbf{i} \equiv \mathbf{i}+4[12]$.

One of our new results is that this theory can be obtained as a slightly unnatural $\mathbb{Z}_{2}$ orbifold of the field theory living on the collection of $N$ self-dual threebranes in Type 0B theory. In that theory we have two $U(N)$ gauge groups, two quadruples of fermions $\psi_{12} \in\left(\overline{\mathbf{N}}_{1}, \mathbf{N}_{2}\right), \quad \psi_{21} \in\left(\overline{\mathbf{N}}_{2}, \mathbf{N}_{1}\right)$, and two sextets of adjoint scalars $\phi_{1}, \phi_{2}$. This theory has a symmetry:

$$
\left(\begin{array}{c}
\phi_{1} \\
\phi_{2}
\end{array}\right) \mapsto\left(\begin{array}{c}
-\phi_{1} \\
-\phi_{2}
\end{array}\right), \quad\left(\begin{array}{c}
\psi_{12} \\
\psi_{21}
\end{array}\right) \mapsto\left(\begin{array}{c}
-\psi_{12} \\
\psi_{21}
\end{array}\right)
$$

Indeed the Yukawa couplings are of the form $\phi_{1} \psi_{12} \psi_{21}$ and $\phi_{2} \psi_{21} \psi_{12}$, hence they are invariant under (2.2). If we add the standard action of $\mathbb{Z}_{2}$ on Chan-Paton indices (i.e. sending $N \rightarrow 2 N$ and conjugating by $\left(\begin{array}{cc}I & 0 \\ 0 & -I\end{array}\right)$ in both of the $U(2 N)$ groups, where $I$ is the $N \times N$ identity matrix) then the resulting theory has the correct field content and the interactions to be identified with the $\mathbb{Z}_{4}$ orbifold of the $\mathcal{N}=4$ theory.

\section{Closed String Theory}

\section{1. $\mathbb{Z}_{4}$ orbifold of Type $\mathbb{I} B$ in the GS formalism}

Let us consider the Type IIB string theory in the Green-Schwarz formalism. In the light cone gauge the action looks like:

$$
S=\int d \tau d \sigma\left(\partial_{+} X^{i} \partial_{-} X^{i}-i S^{a} \partial_{+} S^{a}-i \tilde{S}^{a} \partial_{-} \tilde{S}^{a}\right)
$$

where the fermions $S^{a}, \tilde{S}^{a}$ transform in $\mathbf{8}_{s}$ of $\operatorname{Spin}(8)$, and the scalars $X^{i}$ are in $\mathbf{8}_{v}$. 
Now let us rewrite this action in the form where the $S U(4)$ invariance is manifest. To this end we introduce the complex fermions:

$$
b^{m}=\sqrt{2}\left(S^{2 m}+i S^{2 m-1}\right), \quad \tilde{b}^{m}=\sqrt{2}\left(\tilde{S}^{2 m}+i \tilde{S}^{2 m-1}\right), \quad m=1,2,3,4
$$

The scalars are represented as:

$$
X^{i}=\left(X, \bar{X}, X^{m n}=-X^{n m}\right), \quad m, n=1, \ldots, 4
$$

The action (3.1) is rewritten as:

$$
S=S(X, \bar{X})-i \sum_{m} \int\left(b^{\bar{m}} \partial_{+} b^{m}+\tilde{b}^{\bar{m}} \partial_{-} \tilde{b}^{m}\right)+\frac{1}{4} \sum \epsilon_{m n k l} \partial_{+} X^{k l} \partial_{-} X^{m n}
$$

We wish to perform the orbifold projections with respect to the center of $S U(4)$.

Naturally we get four sectors.

Untwisted sector. In this sector all $X^{i}$ s and $b^{m}, \tilde{b}^{m}$ are integer moded. The zero point energy vanishes both in left and right sectors and the projection of the massless modes gives:

$$
\begin{array}{ll}
S O(8): & \left(\mathbf{8}_{v} \oplus \mathbf{8}_{c}\right) \otimes\left(\mathbf{8}_{v} \oplus \mathbf{8}_{c}\right) \rightarrow \\
S U(4): & \mathbf{6} \otimes \mathbf{6} \bigoplus 4(\mathbf{1} \otimes \mathbf{1}) \bigoplus \mathbf{4} \otimes \overline{\mathbf{4}} \bigoplus \overline{\mathbf{4}} \otimes \mathbf{4}
\end{array}
$$

So, all space-time fermions are projected out, while out of bosons we have got the components of the metric and $B$-field tangent to the fixed plane $(4(\mathbf{1} \otimes \mathbf{1}))$, orthogonal to the fixed plane plus the dilaton $(\mathbf{6} \otimes \mathbf{6})$, and components of the $\mathrm{RR}$ forms which are either transverse or longitudinal with respect to the fixed plane.

Twisted sectors. $X, \bar{X}$ are always integer moded. They contribute $-\frac{1}{12}$ to the zero-point energy both on the left and on the right.

Twists by $\pm i$ :

$$
\begin{aligned}
& X^{m n}(\sigma+2 \pi)=-X^{m n}(\sigma) \\
& b^{m}(\sigma+2 \pi)= \pm i b^{m}(\sigma) \\
& \tilde{b}^{m}(\sigma+2 \pi)= \pm i \tilde{b}^{m}(\sigma)
\end{aligned}
$$

the zero-point energy of the states $| \pm i\rangle_{L, R}$ is given by

$$
E_{L}=E_{R}=-\frac{1}{12}+3\left(-\frac{1}{12}+\frac{1}{2} \frac{1}{2^{2}}\right)+4\left(\frac{1}{12}-\frac{1}{2} \frac{3}{4^{2}}\right)=0
$$

so we get a massless mode for both $+i$ and $-i$ sectors. Later we will see that these states are not scalars but, somewhat surprisingly, the positive and negative helicity components of a photon in $3+1$ dimensions. 
Notice that the next excited levels have $L_{0}=\bar{L}_{0}=\frac{1}{4}$ and transform in the rank two tensor representations of $S U(4)$ :

$$
\begin{aligned}
& b_{-\frac{1}{4}}^{m}|+i\rangle_{L} \otimes \tilde{b}_{-\frac{1}{4}}^{n}|+i\rangle_{R} \in \mathbf{4} \otimes \mathbf{4} \\
& b_{-\frac{1}{4}}^{\bar{m}}|-i\rangle_{L} \otimes \tilde{b}_{-\frac{1}{4}}^{\bar{n}}|-i\rangle_{R} \in \overline{\mathbf{4}} \otimes \overline{\mathbf{4}}
\end{aligned}
$$

Twist by -1 : all bosons are periodic, the fermions are anti-periodic. The zero point energy is negative:

$$
E_{L}=E_{R}=4\left(-\frac{1}{2} \frac{1}{2^{2}}\right)=-\frac{1}{2}
$$

so the ground state $|-1\rangle_{L} \otimes|-1\rangle_{R}$ is tachyonic. In the NSR language this is the tachyon from the $(N S-, N S-)$ sector of Type 0B string. The next level is massless:

$$
S_{-\frac{1}{2}}^{a}|-1\rangle_{L} \otimes \tilde{S}_{-\frac{1}{2}}^{b}|-1\rangle_{R}
$$

and before the projection gives rise to the extra set of $R R$ even forms, just like in Type 0B theory. After the projection it doubles the set of forms we got in the untwisted sector, i.e. we get extra $\mathbf{4} \otimes \overline{\mathbf{4}} \oplus \overline{\mathbf{4}} \otimes \mathbf{4}$.

So, in addition to the $\mathbb{Z}_{2}$ projected bulk modes of Type 0B theory the closed string sector has extra massless modes propagating along the fixed fourplane. We will identify these modes with those of a massless vector field.

\section{2. $\mathbb{Z}_{4}$ orbifold of Type $\mathbb{I} B$ in the NSR formalism}

In the Neveu-Schwarz-Ramond formalism we have only two twisted sectors, as the space-time part of the orbifold group is only $\mathbb{Z}_{2}$, not $\mathbb{Z}_{4}$. Nevertheless, we have effectively the same number of the sectors due to the two different possibilities of the GSO projection: $(-1)^{F}=(-1)^{\tilde{F}}=1$ and $(-1)^{F}=(-1)^{\tilde{F}}=-1$.

It is quite clear that the GS sectors 1 and -1 correspond to the untwisted sector of NSR formalism. The GS sector 1 corresponds to NSR states with $(-1)^{F}=(-1)^{\tilde{F}}=1$, i.e. $(N S+, N S+)$ and $(R+, R+)$, while the GS sector -1 corresponds to NSR states with $(-1)^{F}=(-1)^{\tilde{F}}=-1$, i.e. $(N S-, N S-)$ and $(R-, R-)$. Similarly, the GS sectors $i$ and $-i$ correspond to the -1 twisted sector of NSR with $(-1)^{F}=(-1)^{\tilde{F}}=1$ and $(-1)^{F}=(-1)^{\tilde{F}}=-1$ respectively. Let us see how this works in some detail.

Untwisted sector: In the NS sector we have the following boundary conditions:

$$
\begin{array}{cc}
X^{\mu}(\sigma+2 \pi)=X^{\mu}(\sigma), & X^{M}(\sigma+2 \pi)=X^{M}(\sigma) \\
\psi^{\mu}(\sigma+2 \pi)=-\psi^{\mu}(\sigma), & \psi^{M}(\sigma+2 \pi)=-\psi^{M}(\sigma)
\end{array}
$$


The ground state has $L_{0}=\bar{L}_{0}=-\frac{1}{2}$, which gives the tachyon $T$. This is the state present in the GS -1 sector (3.9). The massless level is generated by

$$
\psi_{-\frac{1}{2}}^{i}|0\rangle_{L} \otimes \tilde{\psi}_{-\frac{1}{2}}^{j}|0\rangle_{R}
$$

which is in $\boldsymbol{8}_{v} \otimes \mathbf{8}_{v}$ of $S O(8)$ and then the projection leaves only the purely transverse or purely longitudinal modes. These are the states we found in the GS untwisted sector (3.5).

In the $\mathrm{R}$ sector both the bosons and the fermions are integer moded, the ground state is supersymmmetric and we have 8 fermion zero modes both in the left and right sectors. In the $(R+, R+)$ sector, which corresponds to the GS untwisted sector, we find states in the $\mathbf{4} \otimes \overline{\mathbf{4}} \bigoplus \overline{\mathbf{4}} \otimes \mathbf{4}$. The same representation is found in the $(R-, R-)$ sector which corresponds to the GS -1 sector.

Twisted sector: the NS boundary conditions are:

$$
\begin{gathered}
X^{\mu}(\sigma+2 \pi)=X^{\mu}(\sigma), \quad X^{M}(\sigma+2 \pi)=-X^{M}(\sigma) \\
\psi^{\mu}(\sigma+2 \pi)=-\psi^{\mu}(\sigma), \quad \psi^{M}(\sigma+2 \pi)=+\psi^{M}(\sigma)
\end{gathered}
$$

The ground state has zero-point energy $L_{0}=\bar{L}_{0}=\frac{1}{4}$, just like the first excited level in the GS description of the $i$ and $-i$ twisted sectors (3.8). The fermions $\psi^{M}$ have zero modes so this excited level is a tensor product of two spinors of $S O(6)$, in agreement with what we wrote in the GS section: in the $(N S+, N S+)$ sector we find the $\mathbf{4} \otimes \mathbf{4}$ of $S U(4)$, as in the GS $i$ sector, in the $(N S-, N S-)$ sector we find the $\overline{\mathbf{4}} \otimes \overline{\mathbf{4}}$ of $S U(4)$, as in the GS $-i$ sector (3.8).

Now, the $\mathrm{R}$ boundary conditions are:

$$
\begin{array}{cc}
X^{\mu}(\sigma+2 \pi)=X^{\mu}(\sigma), & X^{M}(\sigma+2 \pi)=-X^{M}(\sigma) \\
\psi^{\mu}(\sigma+2 \pi)=+\psi^{\mu}(\sigma), & \psi^{M}(\sigma+2 \pi)=-\psi^{M}(\sigma)
\end{array}
$$

The zero point energy vanishes and $\psi^{\mu}$ have zero modes so that both for the left and right movers we find spinors in $3+1$ dimensions. In the $(R+, R+)$ sector the ground state transforms as a product of two positive chirality Weyl spinors: it is a photon of positive helicity (this is the state that corresponds to the ground state of the GS $i$ sector). In the $(R-, R-)$ sector the ground state transforms as a product of two negative chirality Weyl spinors: it is a photon of negative helicity corresponding to the ground state of the GS $-i$ sector.

The above construction of the NSR spectrum is identical to what would be done to describe the $\mathbb{Z}_{2}$ orbifold of Type $0 \mathrm{~B}$ theory by reflection of 6 of the coordinates. Thus, the $\mathbb{Z}_{4}$ orbifold of Type IIB may indeed be thought of as a $\mathbb{Z}_{2}$ orbifold of Type 0B. The bulk 
states of this theory are simply the bulk states of Type 0B invariant under the reflection of 6 of the coordinates. In addition, we find a $3+1$ dimensional massless vector field which lives on the fixed plane $\mathbb{R}^{3,1}$. This effect is somewhat analogous to what happens in the IIB string on ALE singularity where we find a tensor theory on the fixed plane $\mathbb{R}^{5,1}$.

Are there any objects charged with respect to this vector field? In the conventional string theory the quanta of the $U(1)$ field are created by the vertex operator $\mathcal{V}$ of dimension $(1,1)$. It can be written in the canonical superghost picture as follows. Introduce the algebra of the Ashkin-Teller twist operators which create the square root cuts for the coordinates $X^{i}[16,17]$ :

$$
\begin{aligned}
\partial X^{i}(z) \sigma_{j}(0) & =\frac{\delta_{j}^{i}}{z^{\frac{1}{2}}} \tau^{j}(0) \\
\partial X^{i}(z) \tau^{j}(0) & =\frac{\delta^{i j}}{2 z^{\frac{3}{2}}} \sigma_{j}(0)+\frac{2 \delta^{i j}}{z^{\frac{1}{2}}} \partial \sigma_{j}(0)
\end{aligned}
$$

Then the operator $\mathcal{V}$ is

$$
\mathcal{V}=\frac{1}{2} F_{\mu \nu}(k)\left(\gamma^{\mu} \gamma^{\nu}\right)^{\alpha \beta} V_{(-1 / 2) \alpha} \tilde{V}_{(-1 / 2) \beta}
$$

where the chiral operator $V_{(-1 / 2) \alpha}$ of dimension 1 is given by:

$$
V_{(-1 / 2) \alpha}(z)=e^{-\varphi(z) / 2} \Sigma_{\alpha}(z) \prod_{M=4}^{9} \sigma_{M}(z) e^{i k_{\mu} X^{\mu}(z)}
$$

with $\varphi$ being the bosonized superghost and $\Sigma_{\alpha}$ the product of twist operators for the fermions $\psi^{\mu}$ (i.e. $\Sigma_{\alpha}$ is a spinor with respect to the unbroken part $S O(1,3)$ of the Lorentz group). The fact that the sum of the dimensions of all the twist field including that of superghosts is precisely one is a consistency check of the computation (3.7).

We see that it is the field strength, not the gauge field itself, which enters the vertex operator (3.16). This is hardly a surprize given the R-R origin of the $U(1)$ vector field. Therefore we expect that the only way the $U(1)$ field may couple to massless open string states on D-branes is through some kind of a magnetic moment interaction. On the other hand, it is well-known that the D-branes couple to the R-R fields as the elementary charges do.2 Can we find the charged states using D-branes?

First of all, near the fixed plane in the presence of the large number of the D3branes the geometry looks like $A d S_{5} \times \mathbb{R}^{5}$. Since the $U(1)$ field comes from the twisted $(R, R)$ sector, the natural candidates for the charged particles are the 'fractional' D-branes wrapped over various cycles of $\mathbb{R I P}_{5} \cdot 3$ We hope to return to this issue in the future.

2 The point is [18] that in computing the one-point disc amplitude the R-R vertex operator must be written in the $(-2)$ ghost picture while (3.16) has only $(-1)$ unit of the total ghost charge. Upon acting on the operator analogous to (3.16) by the inverse of the picture changing operator $e^{\varphi} G_{0}$ one maps the field strength into the gauge potential.

3 We are grateful to M. Douglas for suggesting this to us. 


\section{Open string theory}

Consider the setup of the previous section and add $N$ D3-branes parallel to the fixed fourplane.

\subsection{Gauge theory on the branes in more detail}

It is a standard by now excersize using the techniques of [4] to work out the field content of the low-energy effective field theory on the stack of D3-branes. Following [6] we get the fields of the four dimensional $U(4 N) \mathcal{N}=4$ gauge theory subject to a certain invariance condition:

$$
h \cdot \Psi^{I g \mid J g^{\prime}}=\Psi^{I g h^{-1} \mid J g^{\prime} h^{-1}}
$$

where $\Psi$ denotes any field of the $U(4 N)$ theory, $h, g, g^{\prime} \in \mathbb{Z}_{4}$, the pair $I g, I=1, \ldots, N$ is a way to enumerate all $4 N$ color indices of the $U(4 N)$ theory, finally $h \cdot \Psi$ denotes the field $\Psi$ transformed by an element $h$ of the R-symmetry group $S U(4)$. In particular, the gauge gluons $A_{\mu}$ are singlets under $h=i^{k}, k=0,1,2,3: h \cdot A_{\mu}=A_{\mu}$, the fermions transform as: $h \cdot \psi_{\alpha}^{m}=i^{k} \psi_{\alpha}^{m}$, the scalars transform as: $h \cdot \phi^{m n}=i^{2 k} \phi^{m n}$. The solution of the equations (4.1) yields the following theory: the gauge group is $G=\times_{\mathbf{i}=0}^{3} U\left(N_{\mathbf{i}}\right), N_{\mathbf{i}}=N$, the global symmetry group is $H=S U(4)$, the fermions transform in $\left(\overline{\mathbf{N}}_{\mathbf{i}+1}, \mathbf{N}_{\mathbf{i}}\right) \otimes \mathbf{4}$ of $G \times H$, the scalars are in $\left(\overline{\mathbf{N}}_{\mathbf{i}+2}, \mathbf{N}_{\mathbf{i}}\right) \otimes \mathbf{6}, \mathbf{i} \equiv \mathbf{i}+4$.

The scalars have a tree level potential $V(\phi)$. Let $\phi_{\mathbf{i}}^{M}$ denote the scalar in $\left(\overline{\mathbf{N}}_{\mathbf{i}+2}, \mathbf{N}_{\mathbf{i}}\right)$,

$M \in \mathbf{6}$ of $H$. The scalars obey a reality condition: $\phi_{\mathbf{i}}^{M, \dagger}=\phi_{\mathbf{i}+2}^{M}$. The potential has the form:

$$
V(\phi)=\sum_{M<N} \operatorname{Tr}\left(\phi_{1}^{M} \phi_{3}^{N}-\phi_{1}^{M} \phi_{3}^{N}\right)^{2}+\operatorname{Tr}\left(\phi_{2}^{M} \phi_{0}^{N}-\phi_{2}^{N} \phi_{0}^{M}\right)^{2}
$$

In addition, there are Yukawa couplings. Let $\psi_{\mathbf{i}}^{m}$ denote the fermion in $\left(\overline{\mathbf{N}}_{\mathbf{i}+1}, \mathbf{N}_{\mathbf{i}}\right), m \in \mathbf{4}$ of $H$. Identify $\mathbf{6}$ of $H$ with $\Lambda^{2} \mathbf{4}: M \leftrightarrow[m n]=-[n m]$. Then the yukawas are:

$$
Y=\sum_{m, n, k, l} \operatorname{Tr} \phi_{2}^{k l}\left(\varepsilon_{k l m n} \psi_{1}^{m} \psi_{0}^{n}-\bar{\psi}_{3}^{\bar{l}} \bar{\psi}_{2}^{\bar{k}}\right)+\text { cyclic }
$$

We shall now identify these fields with the specific sectors in the open string theory keeping in mind further discussion of the interactions of the bulk modes with the modes propagating on the branes. 


\subsection{Vertex operators in the NSR formalism}

We work in the NSR formalism and start with D3-branes placed near, but not exactly at the fixed plane. The plane at $\mathbb{R}^{1,3} \times \mathbb{R}^{6} / \mathbb{Z}_{2}$ parallel to $\mathbb{R}^{1,3}$ lifts to two planes on the covering space, one at $X^{M}=x^{M}$, another at $X^{M}=-x^{M}$. In addition we may have electric and magnetic types of branes. If we start with the configuration of the $N$ self-dual branes, then they lift to the $4 N$ branes on the covering space, $N$ electric and $N$ magnetic branes at $X^{M}=x^{M}$ and $N$ electric and $N$ magnetic branes at $X^{M}=-x^{M}$. Hence we get four types of the endpoints for the open strings: $\left( \pm x^{M}\right)$, e or $\mathbf{m}$. From now on we assume that $x^{M}=0$.

Let $I, J=1, \ldots, N$ denote the Chan-Paton indices. The total of $4 N$ labels are organized in pairs $(I g), I=1, \ldots, N, g=1, i,-1,-i$. In Type IIB string theory the states corresponding to the vector bosons $W^{I g \mid J g^{\prime}}$ propagating with the (matrix) polarization vector $A_{\mu}^{I g \mid J g^{\prime}}(k)$ and the momentum $k_{\mu}$ along the $4 N$ coinciding D-branes are created by the boundary vertex operators which can be written in both $(-1)$ and 0 pictures:

$$
\begin{aligned}
V_{-1}(A, k) & =e^{-\varphi}(A \cdot \psi) e^{i k \cdot X} \\
V_{0}(A, k) & =(A \cdot \partial X+i(k \cdot \psi)(A \cdot \psi)) e^{i k \cdot X}
\end{aligned}
$$

(recall that $A$ 's are matrices!) One can also write down the formulae for the scalar modes. Using the doubling trick (see e.g. [19]) one gets the same formulae as (4.4) except that now the polarization matrix (denoted by $\phi$ ) points in the $M$-direction. Finally, we need the formulae for the vertex operators describing the emission of the spinor states with the wavefunction $\lambda_{I g \mid J g^{\prime}}^{\alpha m}$ :

$$
\begin{aligned}
V_{-1}(\lambda, k) & =e^{-\varphi / 2}\left(\lambda^{\alpha m} \Sigma_{\alpha} \Sigma_{m}\right) e^{i k \cdot X} \\
V_{0}(\lambda, k)_{I g \mid J g^{\prime}} & =e^{\varphi / 2} \lambda^{\beta n} \Gamma_{i, \beta n}^{\alpha m} \Sigma_{\alpha} \Sigma_{m}\left(\partial X^{i}+i(k \cdot \psi) \psi^{i}\right) e^{i k \cdot X}
\end{aligned}
$$

What remains is to impose the $\mathbb{Z}_{4}$ invariance conditions. It amounts to demanding:

$$
\begin{aligned}
A_{\mu, I g \mid J g^{\prime}} & =\sum_{\mathbf{i}=0}^{3} A_{\mu, \mathbf{i}}^{I J} \delta_{g, g^{\prime}} g^{\mathbf{i}} \\
\lambda_{\alpha m, I g \mid J g^{\prime}} & =\sum_{\mathbf{i}=0}^{3} \lambda_{\mu, \mathbf{i}, \mathbf{i}+1}^{I J} \delta_{g, i g^{\prime}} g^{\mathbf{i}} \\
\phi_{M, I g \mid J g^{\prime}} & =\sum_{\mathbf{i}=0}^{3} \phi_{M, \mathbf{i}, \mathbf{i}+2}^{I J} \delta_{g,-g^{\prime}} g^{\mathbf{i}}
\end{aligned}
$$

where the subscripts $\mathbf{i}, \mathbf{i}+2$ etc. indicate the labels of the gauge factors under which the Chan-Paton factors $I, J$ respectively transform.

We see that in considering the coupling of the twisted sector bulk mode to the open string states propagating on the brane we don't need to alter the $\left(-\frac{1}{2},-\frac{1}{2}\right)$ picture of the vertex operator (3.16). Hence the only coupling we might see would be of the magnetic moment type, i.e. with the field strength of the $U(1)$ field on the fixed plane. 


\subsection{Going away from the singularity}

Let us look at the (classical) vacua of our theory. The minima of the potential (4.2) are the solutions to the equations:

$$
\phi_{\mathbf{i}}^{M} \phi_{\mathbf{i}+2}^{N}=\phi_{\mathbf{i}}^{N} \phi_{\mathbf{i}+2}^{M}
$$

By separating the electric stack from the magnetic stack we make all the bifundamental fields massive. This separation corresponds to the solution of (4.7) of the form:

$$
\Phi_{0}^{M N} \equiv \phi_{0}^{M} \phi_{0}^{N, \dagger}=x^{M} x^{N} \mathbf{1}, \quad \Phi_{1}^{M N} \equiv \phi_{1}^{M} \phi_{1}^{N, \dagger}=y^{M} y^{N} \mathbf{1}
$$

where $x^{M} \sim-x^{M} \neq 0, y^{M} \sim-y^{M} \neq 0$ are the coordinates of the separated stacks. A $^{-}$This vev breaks the gauge group down to the $U(N) \times U(N)$ subgroup, where $U(N)$ 's are the diagonal subgroups in the $U\left(N_{0}\right) \times U\left(N_{2}\right)$ and $U\left(N_{1}\right) \times U\left(N_{3}\right)$ respectively. The fields $\phi_{0}^{M}=\phi_{2}^{M}$ and $\phi_{1}^{N}=\phi_{3}^{N}$ act as the six massless adjoint scalars of the first and second $U(N)$ respectively. Thus, as expected, the $\mathbb{Z}_{4}$ theory Higgses down to the theory on the electric and magnetic branes of Type 0B theory at a smooth point (away from the singularity). Indeed, in [8] a stack of electric D3-branes was considered and it was shown that its world volume is described by $S U(N)$ gauge theory coupled to 6 adjoint scalars. The same theory describes a magnetic stack.

\section{Discussion}

In this paper we have considered an orbifold of Type IIB string theory by reflection of six of the coordinates. Because of the action on the fermions this is actually a $\mathbb{Z}_{4}$ orbifold of Type IIB which can equivalently be thought of as a $\mathbb{Z}_{2}$ orbifold of Type 0B string theory. Consideration of the closed strings in the twisted sector led us to conclude that the only massless field on the fixed fourplane $\mathbb{R}^{3,1}$ is a photon. Thus, we have found a simple way of embedding a $U(1)$ gauge theory into string theory.

In view of recent progress on connections between large $N$ gauge theories and strings, it is even more interesting to find an embedding of pure glue $U(N)$ gauge theory into a string theory which might help in searching for its stringy dual. The pure glue $U(N)$

4 A more general configuration is to also separate the branes within each stack so that the bifundamental fields get unequal masses and the gauge group is broken down to the maximal Cartan subgroup. This configuration corresponds to the diagonal matrices $\Phi_{0,1}^{M N}$ with unequal entries. For such a configuration we do not find a non-abelian theory in the infrared. It is a subtle question whether the dynamics favors complete separation of the branes [20]. 
gauge theory may be constructed as a $\mathbb{Z}_{4}$ orbifold of the $\mathcal{N}=4$ supersymmetric $U(N)$ gauge theory by the center of the $S U(4)$ R-symmetry which acts trivially on the group indices. This is known as an 'irregular' orbifold theory because the gauge group twists do not satisfy the condition $\operatorname{Tr} \gamma=0$. If we think of the 4 basic 'fractional' D3-branes of Type $\mathbb{I B}$ on $\mathbb{R}^{6} / \mathbb{Z}_{2}$ as corresponding to the 4 different nodes of the quiver diagram, then the pure glue theory results on a stack of $N$ 'fractional' D3-branes of the same type. The absence of scalar fields in this theory is related to the fact that the fractional branes can exist only on the fixed fourplane and hence cannot fluctuate away from it. Such a stack has non-vanishing tadpoles for the twisted states, and in particular for the tachyon. Thus, as for the stack of electric D3-branes, the dual gravity background will have a radially varying tachyon field [8,21,22]. We postpone a study of this background for the future.

We have also initiated a study of interactions between the photons on the fixed fourplane with the gauge theories on the D3-branes. Overall, this orbifold is quite different from the $\mathcal{N}=2 \mathbb{Z}_{2}$ orbifold studied in $[4$. In that case the scalars on the fixed sixplane could be used to blow up the $\mathbb{R}^{4} / \mathbb{Z}_{2}$ into a smooth ALE space. For $\mathbb{R}^{6} / \mathbb{Z}_{2}$ we find no scalars in the twisted sector, hence it is not clear how to resolve the singularity.

The original motivation for our work was to find a D-brane realization of the orbifold of $\mathcal{N}=4$ SYM by the $\mathbb{Z}_{4}$ center of its $S U(4)$ R-symmetry. We have found such a realization in terms of D3-branes of Type 0B string theory orbifolded by reflection of six coordinates. In general, it is quite clear that if the orbifold group $\Gamma$ is a subgroup of $S O(6)$ then the gauge theory may be realized on D3-branes of Type IIB theory, but if $\Gamma$ also includes the center of $S U(4)$ then the realization involves the D3-branes of Type 0B theory.

\section{Appendix A. Another $\mathbb{Z}_{4}$ orbifold of Type IIB string}

It may be interesting to consider orbifolding by reflection of two of the coordinates:

$$
X^{M} \rightarrow-X^{M}, \quad M=8,9 .
$$

This case is similar to the reflection of 6 coordinates in that it is a $\mathbb{Z}_{4}$ operation on Type IIB string theory. Similarly, it can be thought of as a $\mathbb{Z}_{2}$ orbifold of Type 0B theory. This theory has an $S O(2)$ internal symmetry group and $S O(6)$ transverse group: so compared to the orbifold (2.1) the two are interchanged.

The discussion of the spectrum proceeds analogously to the case (2.1). The NSR untwisted sector gives states of Type 0B theory invariant under (A.1). Something inter-

esting happens in the twisted sector which is localized on the fixed eightplane $\mathbb{R}^{7,1}$. The $(N S, N S)$ zero-point energy is

$$
E_{L}=E_{R}=-1 / 4,
$$


so it seems that there is a tachyon living on the fixed plane. However, the ground state transforms as a vector $A_{M}$ under the $S O(2)$ rotation, hence it is projected out!

Thus, the only massless twisted sector states come from the RR sector. From the $(R+, R+)$ sector we find $\mathbf{4} \otimes \mathbf{4}$ of $S U(4)$, which gives a 1 -form and a selfdual 3 -form of $S O(6)$. From the $(R-, R-)$ sector we find $\overline{\mathbf{4}} \otimes \overline{\mathbf{4}}$ of $S U(4)$, which gives a 1 -form and an anti-selfdual 3-form of $S O(6)$. Putting them together we thus find the gauge potentials, $A_{\mu}, B_{\mu}$ and $A_{\mu \nu \lambda}$ which correspond to two 2-form field strengths and one 4-form field strength. Dualizing one of the 2 -forms we have $F_{2}, F_{4}$ and $F_{6}$. These naturally couple to a 0-brane, a 2-brane and a 4-brane in 8 dimensions. It seems plausible that these objects come from 1-branes, 3-branes and 5-branes wrapped over the $\mathbb{R I}^{1} \approx \mathbf{S}^{1}$ which is the base of the cone. This 8-dimensional theory deserves further study.

\section{Acknowledgements}

We are grateful to O. Bergman, M. Douglas, A. Losev and L. Paniak for useful discussions. The work of I. R. K. was supported in part by the NSF grant PHY-9802484 and by the James S. McDonnell Foundation Grant No. 91-48; that of N. A. N by Harvard Society of Fellows, partly by NSF under the grants PHY94-07194, PHY-98-02709, partly by RFFI under grant 98-01-00327, partly by the grant 96-15-96455 for scientific schools. Research of S. L. Sh. is supported by DOE grant DE-FG02-92ER40704, by NSF CAREER award, by OJI award from DOE and by Alfred P. Sloan foundation. N. A. N. is grateful to ITP, Santa Barbara and to ESI, Vienna for hospitality while some parts of this work were being done. 


\section{References}

[1] J. Maldacena, "The Large N limit of superconformal field theories and supergravity," Adv. Theor. Math. Phys.2 (1998) 231, hep-th/9711200

[2] S.S. Gubser, I.R. Klebanov, and A.M. Polyakov, "Gauge theory correlators from noncritical string theory," Phys. Lett. 428B (1998) 105, hep-th/9802109

[3] E. Witten, "Anti-de Sitter space and holography," Adv. Theor. Math. Phys.2 (1998) 253 , hep-th/9802150

[4] M. Douglas and G. Moore, "D-branes, quivers, and ALE instantons," hep-th/9603167

[5] S. Kachru and E. Silverstein, "4d conformal field theories and strings on orbifolds," Phys. Rev. Lett. 80 (1998) 4855, hep-th/9802183.

[6] A. Lawrence, N. Nekrasov and C. Vafa, "On conformal field theories in four dimensions," Nucl. Phys. B533 (1998) 199, hep-th/9803015

[7] L. Dixon and J. Harvey, "String theories in ten dimensions without space-time supersymmetry", Nucl. Phys. B274 (1986) 93 ;

N. Seiberg and E. Witten, "Spin structures in string theory", Nucl. Phys. B276 (1986) 272 ;

C. Thorn, unpublished

[8] I. R. Klebanov and A. A. Tseytlin, "D-Branes and Dual Gauge Theories in Type 0 Strings," Nucl. Phys. B546 (1999) 155, hep-th/9811035

[9] A.M. Polyakov, "The Wall of the Cave," hep-th/9809057

[10] O. Bergman and M. Gaberdiel, "A Non-supersymmetric Open String Theory and S-Duality," Nucl. Phys. B499 (1997) 183, hep-th/9701137

[11] I. R. Klebanov and A. A. Tseytlin, "Non-supersymmemtric CFT from Type 0 String Theory," JHEP 9903(1999) 015, hep-th/9901101;

I. R. Klebanov, "Tachyon Stabilization in the AdS/CFT Correspondence," hepth/9906220

[12] N. Nekrasov and S. Shatashvili, "On non-supersymmetric CFT in four dimensions," hep-th/9902110., L. Okun Festschrift, North-Holland, in press

[13] M. Bershadsky, Z. Kakushadze and C. Vafa, "String expansion as large N expansion of gauge theories", Nucl. Phys. B523 (1998) 59, hep-th/9803076

[14] R. Blumenhagen, A. Font and D. Lust, "Non-Supersymmetric Gauge Theories from D-Branes in Type 0 String Theory," hep-th/9906101.

[15] M. Billó, B. Craps and F. Roose, "On D-branes in Type 0 String Theory," hepth/9902196.

[16] L. Dixon, D. Friedan, E. Martinec and S. Shenker, "The Conformal Field Theory of Orbifolds," Nucl. Phys. B282 (1987) 13

[17] M. Bershadsky and A. Radul, "Conformal Field Theories with Additional $\mathbb{Z}_{N}$ Symmetry," Int. J. Mod. Phys. A2 (1987) 165 
[18] J. Polchinski, "TASI Lectures on D-Branes", hep-th/9611050

[19] I. R. Klebanov and A. Hashimoto, "Scattering of Strings from D-branes," hep$\operatorname{th} / 9611214$

[20] K. Zarembo, "Coleman-Weinberg Mechanism and Interaction of D3-branes in Type 0 String Theory, hep-th/9901106

[21] J. Minahan, "Glueball Mass Spectra and Other Issues for Supergravity Duals of QCD Models," hep-th/9811156

[22] I.R. Klebanov and A.A. Tseytlin, "Asymptotic Freedom and Infrared Behavior in the Type 0 String Approach to Gauge Theory," Nucl. Phys. B547 (1999) 143, hepth/9812089 